\title{
Residual errors in visuomotor adaptation persist despite extended motor preparation periods
}

\author{
Authors: Matthew Weightman ${ }^{1,3,4^{*}}$, John-Stuart Brittain ${ }^{2,4}$, R. Chris Miall ${ }^{2,3,4}$, Ned \\ Jenkinson ${ }^{1,3,4}$
}

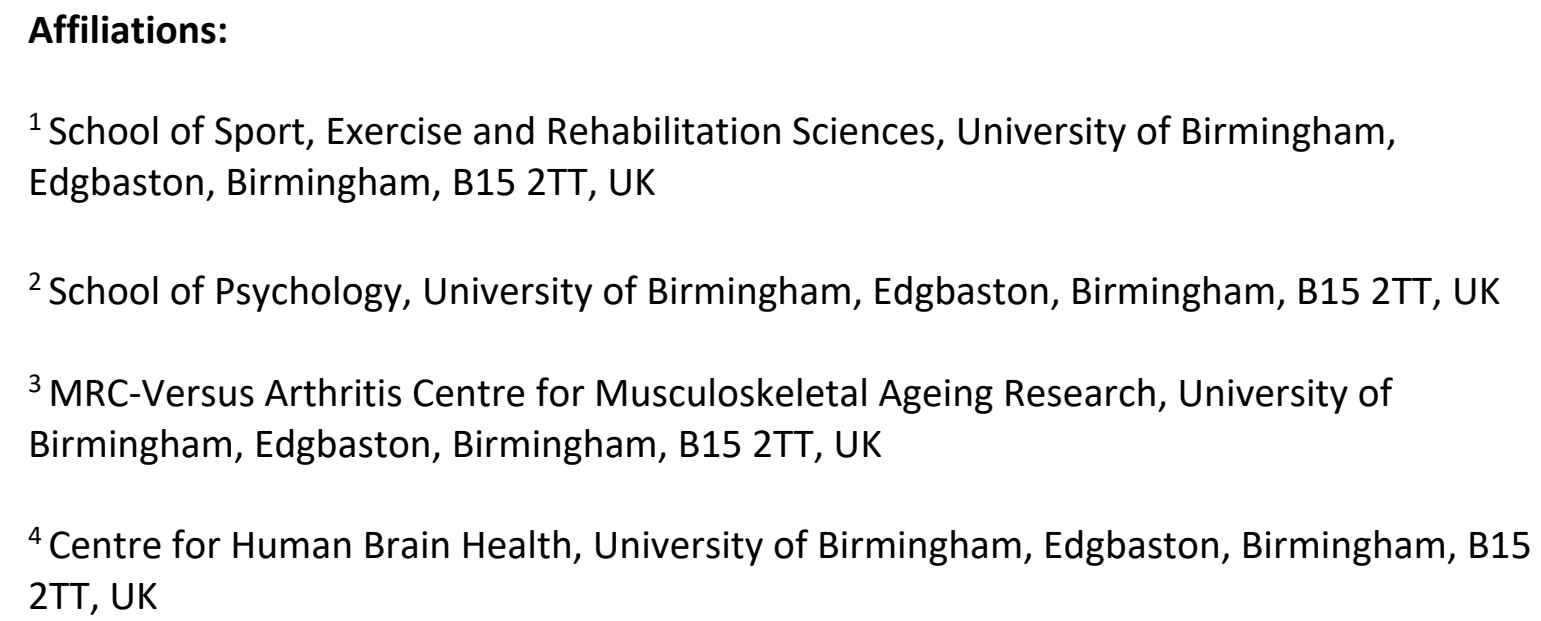

*Corresponding Author:

Matthew Weightman

School of Sport, Exercise and Rehabilitation Sciences,

The University of Birmingham

Edgbaston

Birmingham

B15 2TT

Email: mcw423@adf.bham.ac.uk

Running Head: Persistent visuomotor errors despite extended motor preparation 


\section{Abstract}

44 A consistent finding in sensorimotor adaptation is a persistent undershoot of full compensation, such that performance asymptotes with residual errors greater than seen at baseline. This behaviour has been attributed to limiting factors within the implicit adaptation system, which reaches a sub-optimal equilibrium between trial-by-trial learning and forgetting. However, recent research has suggested that allowing longer motor planning periods prior to movement eliminates these residual errors. The additional planning time allows required cognitive processes to be completed before movement onset, thus increasing accuracy. Here we looked to extend these findings by investigating the relationship between increased motor preparation time and the size of imposed visuomotor rotation $\left(30^{\circ}, 45^{\circ}\right.$ or $\left.60^{\circ}\right)$, with regards to the final asymptotic level of adaptation. We found that restricting preparation time to 0.35 seconds impaired adaptation for moderate and larger rotations, resulting in larger residual errors compared to groups with additional preparation time. However, we found that even extended preparation time failed to eliminate persistent errors, regardless of magnitude of cursor rotation. Thus, the asymptote of adaptation was significantly less than the degree of imposed rotation, for all experimental groups. Additionally, there was a positive relationship between asymptotic error and implicit retention. These data suggest that a prolonged motor preparation period is insufficient to reliably achieve complete adaptation and therefore our results provide support for the proposal that the balance between error-based learning and forgetting (i.e., incomplete retention) contributes to asymptotic adaptation levels. 


\section{Introduction}

70

71

72

Sensorimotor adaptation has been extensively studied using visuomotor rotations

(Cunningham, 1989; Krakauer, Ghilardi, \& Ghez, 1999; Krakauer, Pine, Ghilardi, \& Ghez, 2000). In this task, individuals adapt reaching movements to counter visual feedback that is rotated, for example, $30^{\circ}$ or $45^{\circ}$ from the hand's position. However, adaptation is typically incomplete, and performance plateaus with errors a few degrees greater than at baseline, regardless of the rotation magnitude imposed (Fernandez-Ruiz, Wong, Armstrong, \& Flanagan, 2011; Huberdeau, Haith, \& Krakauer, 2015; van der Kooij, Brenner, van Beers, \& Smeets, 2015; Vaswani et al., 2015; Weightman, Brittain, Punt, Miall, \& Jenkinson, 2020; Langsdorf, Maresch, Hegele, McDougle, \& Schween, 2021). State-space models of learning accurately capture this incomplete compensation (Smith, Ghazizadeh, \& Shadmehr, 2006; Kording, Tenenbaum, \& Shadmehr, 2007). In these models, error-driven learning and forgetting (or a reversion to baseline) work in opposition, and equilibrate below optimal performance, resulting in the commonly observed persistent error. However, Vaswani and colleagues (2015) demonstrated that under certain conditions, individuals disengage from this limited error-dependent learning to attain greater task success. In one of their experimental conditions, Vaswani et al. (2015) 'clamped' visual feedback after an initial learning block, such that a small, fixed, visual error was presented, regardless of movement accuracy. They found that under these conditions, participants appeared to select an alternative, more exploratory learning policy, which enabled them to close the errors and even to overcompensate for the rotation (which was not predicted by the state-space model). The authors suggested that the altered feedback distribution triggered alternative learning processes (and enabled the elimination of residual errors), which are usually suppressed during naturalistic feedback conditions. 
under normal, non-clamped environments, the central nervous system fails to eliminate

residual errors during motor adaptation. They suggested an intrinsic speed-accuracy trade-

off: where time consuming planning processes are interrupted by the imperative onset of

movement, resulting in fast, but inaccurate movements. In their study, they showed that

when an obligatory 2.5 second wait period was introduced between target presentation and

movement initiation, participants were able to fully adapt to a $45^{\circ}$ rotation, leaving no

persisting errors at the end of learning. However, if this wait period was not enforced or was

introduced at the end of the movement, when no planning was assumed to be taking place,

participants failed to fully counteract the rotation. The authors highlighted mental rotation

as a time-consuming cognitive process potentially involved in the planning of visuomotor adaptation.

For some time now, visuomotor adaptation has been framed as a combination of distinct learning processes (Mazzoni \& Krakauer, 2006; Taylor, Krakauer, \& Ivry, 2014; Bond \&

112 detected perturbation (Taylor \& Ivry, 2011; Heuer \& Hegele, 2008; McDougle et al., 2015).

113 Such strategies are thought to include a form of metal rotation (McDougle \& Taylor, 2019).

114 Langsdorf et al. (2021) suggest that under naturalistic feedback conditions, parametric 
terminated (despite no time constraints) and result in aimed movement trajectories falling

117 short of the imposed rotation.

118 Behavioural and neurophysiological research suggests a role of mental rotation in the

119 planning of movements aimed at angles away from visually defined targets (Georgopoulos

120 \& Massey, 1987; Bhat \& Sanes, 1998; Georgopoulos, Lurito, Petrides, Schwartz, \& Massey,

121 1989; Lurito, Georgakopoulos, \& Georgopoulos, 1991; Pellizzer \& Georgopoulos, 1993).

122 Neuronal population vectors recorded in the monkey motor cortex gradually rotate from a

123 stimulus direction to a cued movement direction during the planning of a reach

124 (Georgopoulos et al., 1989; Lurito et al., 1991). Additionally, the completion of mental rotation tasks require long reaction times with larger magnitudes of rotation (Shepard \&

127 (Fernandez-Ruiz et al., 2011; Haith, Huberdeau, \& Krakauer, 2015; McDougle \& Taylor, 128 2019).

129 Given this knowledge, we aimed to replicate and extend the findings of Langsdorf et al.

130 (2021), in order to further understand the roles of extended planning periods and mental

131 rotation in attaining full adaptation. We designed an online visuomotor adaptation task,

132 where participants had either long ( 2.5 seconds), medium ( 1 second) or short ( 0.35 seconds)

133 enforced preparation periods between target presentation and movement onset, and were

134 required to adapt to either a small $\left(30^{\circ}\right)$, moderate $\left(45^{\circ}\right)$ or large $\left(60^{\circ}\right)$ visuomotor rotation.

135 Previous studies (Langsdorf et al., 2021) suggest that the $45^{\circ}$ rotation would be fully

136 corrected with the longest preparation period; errors for the middle interval would be

137 greater; and a substantial residual error should be found for the shortest preparation interval. This negative relationship between preparation interval and residual error should 
compensation even at the shortest preparation interval. In other words, the response time

141 could be considered the sum of a mental rotation period linearly related to the rotation magnitude, and a fixed movement initiation period; if the sum exceeds the prescribed preparation interval, then residual errors should be seen.

\section{Materials and Methods}

147 A total of 180 participants were recruited (aged 18-37 years, mean \pm SD $=23 \pm 4$ years, 82 males); all gave informed consent before participating and received no remuneration for completing the study. The study was approved by the University of Birmingham ethics board (Science, Technology, Engineering and Mathematics Ethical Review Committee). preferred hand to complete the task (all but 5 of the left-handed participants completed the task using their right hand). All participants had normal, or corrected to normal, vision and reported no history of neurological disease. Participants were pseudorandomised into one of nine experimental groups (each $n=20$ ), which differed in the amount of preparation time provided $\left(0.35,1\right.$ or 2.5 seconds) and the magnitude of cursor rotation $\left(30^{\circ}, 45^{\circ}\right.$ or $\left.60^{\circ}\right)$.

Task

We developed a visuomotor adaptation task using the behavioural science experiment platform: PsychoPy (Peirce et al., 2019), which was implemented online via Pavlovia (https://pavlovia.org). Participants were required to have access to a desktop or laptop computer with internet connection and a mouse or trackpad to navigate through and complete the task (117 participants used a trackpad; 63 participants used a mouse). 
Participants were sent a link to access the task and entered a unique code which

172 Prior to starting the task, all participants completed a brief tutorial, which included the task instructions and aims. At this point, participants were asked to contact a researcher if they were unclear about any part of the task and/or had any questions relating to the instructions. of the screen (grey background). The cursor was surrounded by two larger red annuli, which formed a bullseye-like formation (Figure. 1b). At a set moment, the outer ring disappeared, followed by the inner ring 0.5 seconds later, and the cursor then changed colour from red to white after a further 0.5 seconds. Participants were told that this sequence of events should be treated as a countdown to movement and that they should aim to time their movement so that it was initiated in synchrony with the cursor changing colour from red to white

183 (Figure. 1b). Depending on the experimental group, the target (green filled circle) would appear either 2.5 seconds, 1 second or 0.35 seconds before the movement cue - thus creating three distinct preparation periods. The target could appear at any one of eight possible locations, equally spaced (separated by $45^{\circ}$ ) around an invisible circular array 
location once every cycle of eight trials. The position at which the cursor passed the invisible movement accuracy. After an inter-trial interval of 0.5 seconds the cursor would reset to the central position ready for the next trial. Participants were encouraged to move their hand back to a comfortable starting position after each trial. All elements of the visual display were scaled according to the size of the task window and thus were the same relative size

194 for all participants. Consequently, the physical movement size required varied between participants depending on their set up and was not recorded beyond input device. If participants attempted to move 0.15 seconds before or after the go cue, or if movement time exceeded 0.25 seconds, the message "Response too fast/slow" appeared on the screen and the trial was aborted. These measures were to encourage fast movements, initiated correctly with the task instructions.
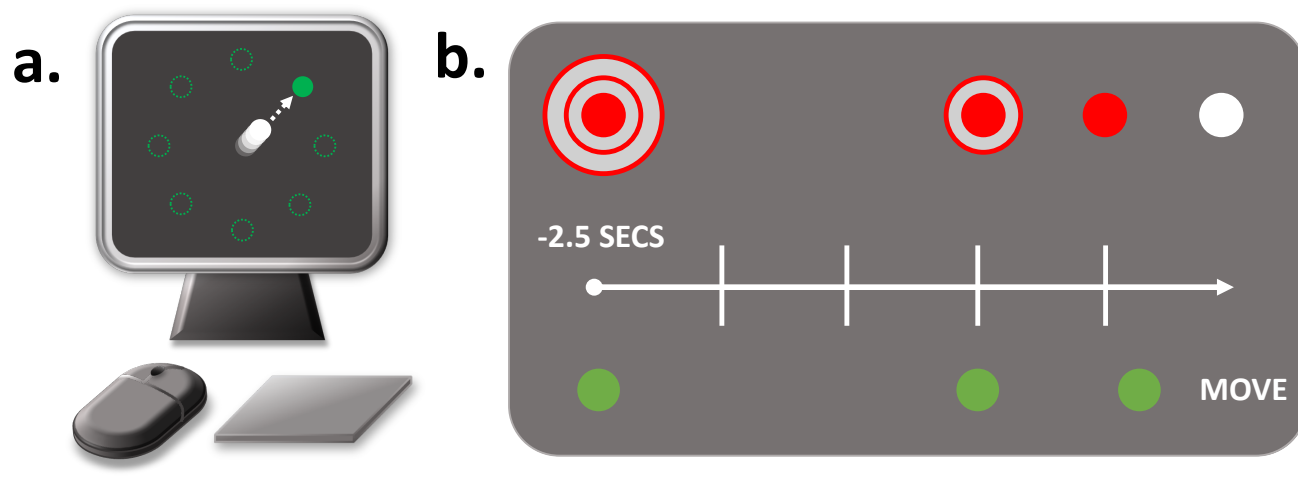

c.

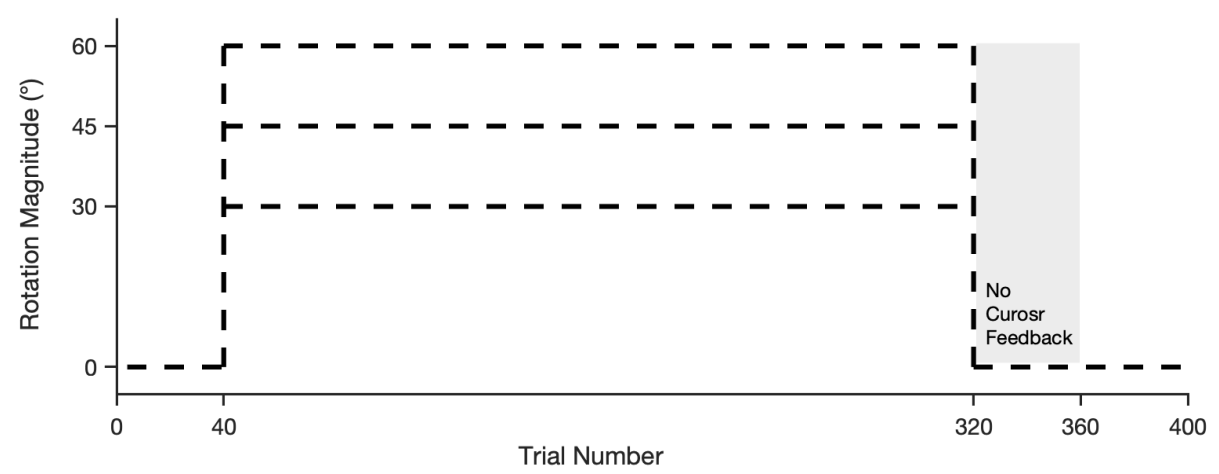

200 Figure 1: The experimental design. a Participants used either a mouse or trackpad to direct 201 an on-screen cursor towards targets presented radially around a central starting position. $\boldsymbol{b}$ 202 An example of the cursor countdown sequence and movement cue. At the beginning of each 
trial the (red) cursor would appear in the centre of the screen, flanked by two larger (red) rings (shown here on the top row). During the trial the two rings would disappear in sequence and the cursor would change colour to white. Participants were required to initiate their movement in synchrony with the cursor colour change, using the disappearing rings as a countdown. The target (shown on the bottom row) would appear either 2.5, 1 or 0.35 seconds prior to the movement cue, depending on group assignment. $c$ Time course of the study protocol. Participants completed 40 baseline trials, followed by 280 adaptation trials (where either $a 30^{\circ}, 45^{\circ}$ or $60^{\circ}$ cursor rotation was imposed), then 40 no-feedback trials and finally 40 de-adaptation trials.

\section{Experimental Design}

Participants first performed eight practice trials (one at each target location). These trials were performed with veridical cursor feedback and were used to familiarise the participants with the timing of their version of the task. The main task directly followed the practice trials and included four phases: baseline, adaptation, no-feedback and de-adaptation (Figure. 1c). During baseline trials $(n=40)$ participants received veridical cursor feedback, such that the on-screen cursor moved in accordance with participants' movements. Adaptation trials immediately followed, in which the cursor feedback was rotated $30^{\circ}, 45^{\circ}$ or $60^{\circ}$ (depending on the experimental group, see below) relative to participants' movement. This block lasted 280 trials, selected to ensure learning approached its asymptotic limit, based on pilot data collected prior to the present study. While there is some indication that full asymptotic saturation of learning was not achieved in all conditions, regression analysis of the last 40 trials of adaptation indicated that there was no significant trend remaining (all $p>0.19$ ) and thus we use the term 'asymptote' to refer to the final state reached at the end of the 280 adaptation trials. Following adaptation trials, participants performed no-feedback trials $(n=40)$. During these trials, participants were told to stop using any strategies they might have employed to achieve the task objectives and try to aim directly for the target. The cursor was hidden at all times during the trial, 

point error were then restored for the final de-adaptation trials $(n=40)$.

throughout the whole task; the rotation was applied only during the adaptation phase.

\begin{tabular}{c|ccc|ccc|ccc} 
& \multicolumn{3}{|c|}{0.35 seconds } & \multicolumn{3}{c|}{1 1 second } & \multicolumn{3}{c}{2.5 seconds } \\
\cline { 2 - 10 } & $30^{\circ}$ & $45^{\circ}$ & $60^{\circ}$ & $30^{\circ}$ & $45^{\circ}$ & $60^{\circ}$ & $30^{\circ}$ & $45^{\circ}$ & $60^{\circ}$ \\
\hline $\mathrm{N}$ & 20 & 20 & 20 & 20 & 20 & 20 & 20 & 20 & 20 \\
(n male) & $(9)$ & $(6)$ & $(8)$ & $(11)$ & $(12)$ & $(4)$ & $(10)$ & $(13)$ & $(9)$ \\
\hline $\begin{array}{c}\text { Age } \\
\text { (years) } \\
\pm \text { SD }\end{array}$ & $\begin{array}{c}23.5 \\
( \pm 2.0)\end{array}$ & $\begin{array}{c}25.4 \\
( \pm 3.4)\end{array}$ & $\begin{array}{c}( \pm 3.9 \\
( \pm 3.4)\end{array}$ & $\begin{array}{c}21.0 \\
( \pm 1.1)\end{array}$ & $\begin{array}{c}21.5 \\
( \pm 3.7)\end{array}$ & $\begin{array}{c}22.6 \\
( \pm 5.0)\end{array}$ & $\begin{array}{c}20.8 \\
( \pm 1.3)\end{array}$ & $\begin{array}{c}21.0 \\
( \pm 1.6)\end{array}$ & $\begin{array}{c}22.7 \\
( \pm 3.7)\end{array}$
\end{tabular}

Table 1: Summary of experimental groups.

241 There were three main outcome variables: reach angle, response time and movement duration. Our primary dependent variable - reach angle - was the angular difference between the target location and participants' movement at end-point (i.e., the difference in angle between the vector linking the starting position and the target marker, and the vector linking the starting position and the point at which participants' movement crossed the

246 target circle perimeter). Trials were deemed outliers and removed if they fell 2.5 standard

247 deviations outside the group average on each trial. A total of $1.06 \%$ of all trials were

248 removed from further analysis. Data from each participant was then averaged into bins of 4 trials to be used for visual representation and statistical analysis. Response time was defined as the time period between target presentation and movement onset (i.e., cursor 
movement duration was defined as the time period between movement onset and when movement crossed the target circle. Reach angle data was not recorded for trials where the response or movement time limits were violated.

Statistical Analysis

Asymptotic levels of adaptation were defined as the mean error over the last 40 trials of the adaptation phase and compared between groups in a two-way ANOVA (Rotation Magnitude $=3$ levels $\left(30^{\circ} / 45^{\circ} / 60^{\circ}\right) \times$ Preparation Time $=3$ levels ( 0.35 seconds $/ 1$ second $/ 2.5$ seconds $)$ ), with any significant main effects or interactions followed up with Bonferrroni-corrected multiple comparisons. Asymptotic differences from the imposed rotation magnitude were compared using a Wilcoxon Signed Rank test, after data from some groups failed normality checks (Shapiro-Wilk test). All statistical analyses were carried out in MATLAB (R2018b) and SPSS (IBM, version 27). All ANOVAs were run as general linear models. The threshold for statistical significance was set at $p<0.05$ and we report $W, F$ and $p$-values, as well as effect sizes from ANOVAs (partial eta squared $\left(n p^{2}\right)$ ).

Modelling

To model the adaptation data, we made two assumptions. First, the time required to plan a reach action was linearly proportional to the rotation magnitude $M$ (i.e., mental rotation speed ( $r s$ ) was constant). If preparation time ( $\mathrm{T}$ ) was insufficient, the planned reach angle was to the angle mentally reached. Second, the asymptotic level of adaptation (achieved reach angle, A) would be proportional to the planned reach angle scaled by the learning ratio (Ir) of learning and forgetting rates (Smith et al., 2006). Thus, 


$$
A=l r^{*} \min \left(M, r s^{*} T\right),
$$

277 with two free parameters, Ir and $r s$. The mean asymptotic reach angles for the 9 groups

were fitted with this equation using the MATLAB ' $n$ linfit' function, estimating $/ r$ and $r s$, and

\section{Results}

\section{Baseline performance does not differ between groups}

Differences in reach angle during the baseline phase were assessed in a two-way ANOVA

Rotation Magnitude $\left(F(2,172)=0.71, p=0.93, n p^{2}=0.001\right)$, Preparation Time $(F(2,172)=$

291 to influence performance later in the task. We felt this analysis important, given the limited experimenter input associated with online research.
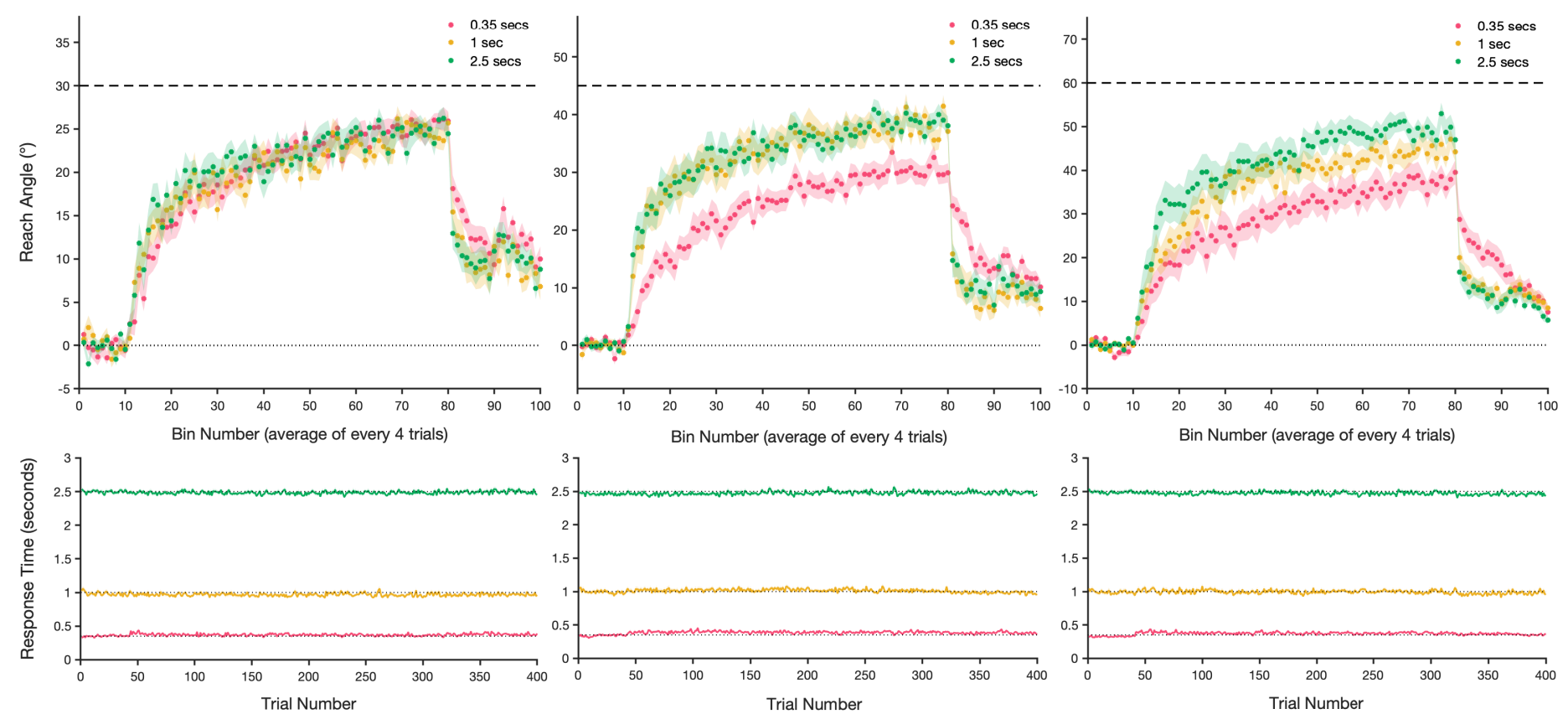
Figure 2: Mean reach angle and response time for each of the experimental groups. Top panel: Reach error ( \pm standard error, shaded region) is averaged every four trials into bins for (from left to right) the $30^{\circ}, 45^{\circ}$ and $60^{\circ}$ groups, with zero degrees and the magnitude of imposed rotation shown as a dotted and dashed line respectively. Bottom panel: Mean response time (time between target presentation and movement initiation), \pm standard error (shaded region) for each group. Note, preparation times were predetermined at $0.35,1$ or 2.5 seconds (dotted lines) and were tightly controlled.

\section{The effect of differing preparation time on asymptotic levels of adaptation}

A two-way ANOVA comparing asymptotic levels of adaptation revealed significant main effects for Rotation Magnitude $\left(F(2,172)=121.01, p<0.001, n p^{2}=0.59\right)$, Preparation Time $\left(\mathrm{F}(2,172)=15.17, \mathrm{p}<0.001, n \mathrm{p}^{2}=0.15\right)$ and a significant Rotation Magnitude*Preparation Time interaction $\left(F(4,172)=5.24, p<0.001, n p^{2}=0.11\right)$. Multiple comparisons revealed that in the $45^{\circ}$ rotation group, there were some differences between learning asymptotes, with regards to preparation time provided. The 0.35 second preparation time group displayed impaired adaptation compared to the 1 and 2.5 second groups (both $p<0.001$ ), with no further differences between the latter two conditions $(p>0.99)$. However, contrary to Langsdorf et al. (2021), all final levels of adaptation significantly differed from $45^{\circ}(0.35$ seconds: $\mathrm{W}=-210.0, \mathrm{p}<0.001,95 \% \mathrm{Cl}=[-16.72,-11.32] ; 1$ second: $\mathrm{W}=-200.0, \mathrm{p}<0.001$, $95 \% \mathrm{Cl}=[-9.71,-2.50] ; 2.5$ seconds: $\mathrm{W}=-210.0, \mathrm{p}<0.001,95 \% \mathrm{Cl}=[-9.33,3.18])$. The 0.35 seconds preparation time group compensated $67.3 \%$ for the rotation, with the 1 and 2.5 second groups achieving $85.1 \%$ and $86.1 \%$ compensation respectively (Figures. $2 \& 3$ ), suggesting that additional preparation time allowed for greater compensation but not the elimination of residual errors.

Similarly, for the $60^{\circ}$ rotation condition, shortening planning time to 0.35 seconds significantly reduced the final level of adaptation compared to both 1 and 2.5 second preparation periods ( 0.35 seconds vs 1 second: $p=0.001,0.35$ seconds vs 2.5 seconds: $p<$ 
0.001). However, there was no difference between the 1 and 2.5 second groups with

321 respect to final adaptation levels $(p=0.17)$. Participants averaged $62.4 \%$ compensation with

3220.35 seconds preparation time, $75.0 \%$ with 1 second preparation time and $81.7 \%$ with 2.5

323 seconds preparation time (Figures. 2 \& 3). Additionally, all asymptotic levels of adaptation

324 significantly differed from the imposed $60^{\circ}$ rotation $(0.35$ seconds: $W=-210, p<0.001,95 \%$

325

$\mathrm{Cl}=[-31.14,-16.02] ; 1$ second: $\mathrm{W}=-210.0, \mathrm{p}<0.00195 \% \mathrm{Cl}=[-16.27,-10.27] ; 2.5$ seconds:

$W=-206.0, p<0.001,95 \% \mathrm{Cl}=[-16.76,-6.44])$.

327 In the $30^{\circ}$ rotation group, there were no differences in asymptotic error between any of

328 the different preparation time groups (all $p>0.99$, Figure. 2). Furthermore, all final levels of adaptation differed significantly from $30^{\circ}(0.35$ seconds: $\mathrm{W}=-210.0, \mathrm{p}<0.001,95 \% \mathrm{Cl}=[-$ and that short periods of preparation interrupt planning, leading the greater residual errors. error, suggesting that mental rotation was completed within the allowed time. But even in of the preparation time provided. 


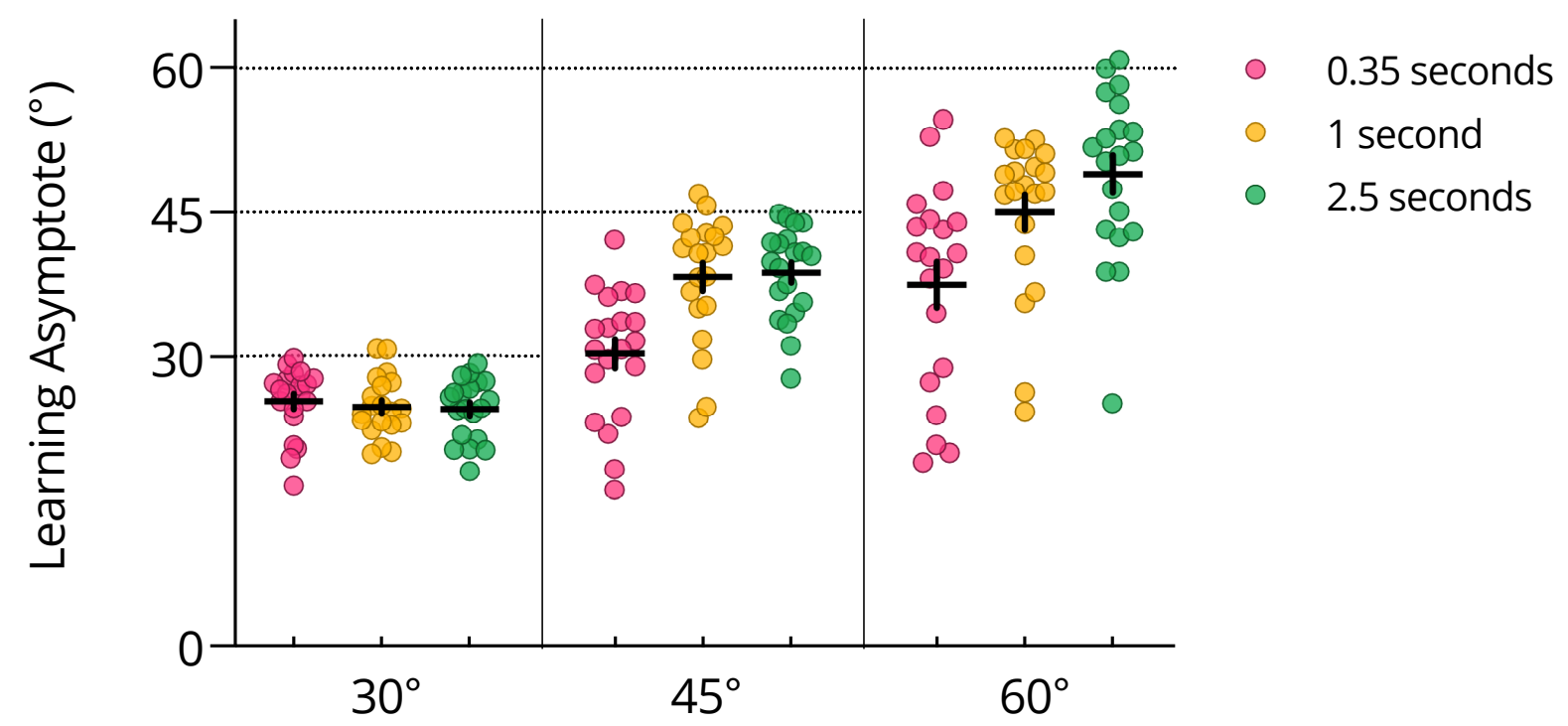

341 Figure 3: The asymptotic levels of adaptation for each participant in each of the rotation 342 magnitude and preparation time groups. Filled circles represent the adaptation asymptote

343 for each participant (defined as the average of the last 40 adaptation trials), with mean 344 values and standard error depicted by horizontal and vertical lines respectively. Dashed horizontal lines depict the imposed cursor rotation for each group.

Modelling asymptotic performance

348 The nine group mean asymptotic reach angles were well fitted by the model (Figure. 4), with

349 the confidence intervals of the fit overlapping with each group mean. The estimated values

350 of the two free parameters were $\mathrm{Ir}=0.814(95 \% \mathrm{Cl}: 0.761-0.867)$ and $r s=118.9$

351 degrees/sec, $(95 \% \mathrm{Cl} 101.64$ - 136.34). From the learning ratio, Ir, the learning rate is

352 estimated to be at least 4.4 times the forgetting rate. This is potentially an underestimate,

353 however, as some of adaptation curves may not have fully saturated (Figure. 2), despite 


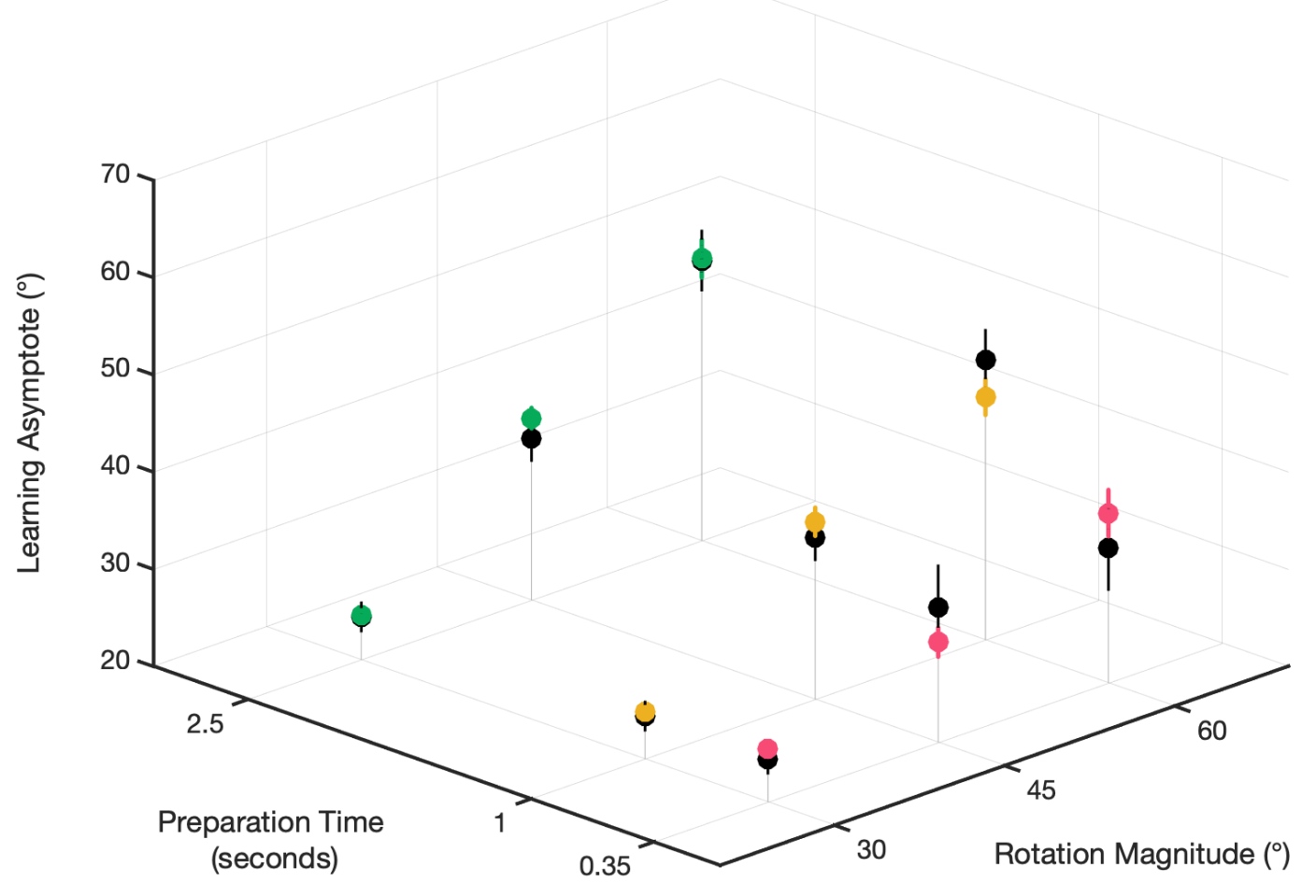

Figure 4. The group mean asymptotic adaptation angles (coloured dots, \pm 1 SEM) are well estimated by the model (see main text). The model (black dots, $\pm 95 \%$ confidence intervals) assumes a fixed mental rotation speed and a fixed ratio between learning and forgetting rates.

\section{Restricting motor preparation time resulted in a stronger implicit retention}

Implicit retention was defined as the mean reach angle in the first 16 no-feedback trials (two cycles of reaches to each target location) and was compared between groups in a twoway ANOVA (Rotation Magnitude x Preparation Time). Results from the ANOVA revealed a significant main effect of Rotation Magnitude $\left(F(2,172)=6.71, p=0.002, \eta p^{2}=0.07\right)$ and Preparation Time $\left(F(2,172)=18.33, p<0.001, \eta p^{2}=0.18\right)$, but no significant interaction $(F$ $\left.(4,172)=1.08, p=0.37, n p^{2}=0.03\right)$. Multiple comparisons showed that participants in the restricted 0.35 second motor preparation time groups displayed greater retention than both the 1 second $(p<0.001)$ and 2.5 second $(p<0.001)$ groups, with no differences between the latter two $(p>0.99)$. 


\section{Input device (trackpad vs mouse) had no effect on late adaptation levels}

373 In order to determine whether the input device used to complete the task affected the

374 extent of adaptation, we compared asymptotic learning levels in each rotation and

375 preparation time group, dependent on whether a mouse or trackpad was used, in a $3 \times 3 \times 2$

376 mixed-design ANOVA (Rotation Magnitude $\mathrm{x}$ Preparation Time $\mathrm{x}$ Input Device). The ANOVA

377 revealed no significant main effect of Input Device $\left(F(1,172)=1.13, p=0.29, n p^{2}=0.007\right)$

378 and no significant three-way interaction $\left(F(4,172)=0.38, p=0.82, n p^{2}=0.009\right)$. In fact, no

379 interaction that included the term Input Device revealed significance (Rotation

380 Magnitude*Input Device: $F(2,172)=0.53, p=0.59, n p^{2}=0.006$, Preparation Time*Input

381 Device: $\left.F(2,172)=0.21, p=0.81, n p^{2}=0.003\right)$, suggesting that the input device used had

382 little effect on adaptive performance during the task (Supplementary Figure. 1, see

383 https://doi.org/10.6084/m9.figshare.14797926).

\section{Discussion}

We aimed to test the assumption that increased motor preparation periods may allow for more complete adaptation during visuomotor rotation tasks. As such, we hypothesised that shorter preparation periods would be sufficient to fully counteract a $30^{\circ}$ rotation, as a small rotation would require less (and therefore quicker) mental rotation before motor execution. We then predicted that this effect would scale with an increased cursor rotation, so that $45^{\circ}$ and $60^{\circ}$ would require greater mental rotation and thus more time to fully compensate. Indeed, we did find that restricting planning time for moderate and larger rotations resulted in impaired final adaptation levels and greater implicit retention compared to those groups 
found no evidence to suggest that extended motor preparation periods allow for the displayed final adaptation performance significantly lower than the imposed cursor rotation. allowed individuals to fully counteract a $45^{\circ}$ visuomotor rotation, leaving no residual mean error at asymptote. The authors suggested that the extended planning period allowed for time-consuming cognitive processes, such as mental rotation, to be completed prior to the go-signal and resulted in complete compensation. This theory, centred around a speedaccuracy trade-off, seems plausible given that reaction times increase in a linear fashion when subjects are asked to reach at increasing angles from a target - a relationship that mimics the behaviour seen in mental rotation tasks (Shepard \& Metzler, 1971;

408 Georgopoulos \& Massey, 1987; McDougle \& Taylor, 2019). Accordingly, brain regions activated in both visuomotor adaptation and mental rotation tasks have also been shown to overlap (Anguera, Reuter-Lorenz, Willingham, \& Seidler, 2010), suggesting that mental rotation contributes to visuomotor adaptation. Recent evidence investigating preparatory

412 activity in the motor cortex of non-human primates further suggests the necessity for motor 413 planning time in visuomotor adaptation (Vyas, O’Shea, Ryu, \& Shenoy, 2020). In addition,

414 Langsdorf et al. (2021) found that delaying movement initiation resulted in an overcompensation of the imposed rotation, similar to the overshoot in reach estimation shown by Georgopoulos and Massey (1987), suggesting similar cognitive rotation strategies may have been in play. 
Notwithstanding these studies, we found that on average participants' learning plateaued

before eliminating error, with asymptotic learning levels statistically different from the

420 degree of imposed rotation for all rotational groups $\left(30^{\circ}, 45^{\circ}, 60^{\circ}\right)$, regardless of the amount

421 of preparation time enforced. These findings are consistent with previous reports which

422 have suggested that sustained errors during visuomotor adaptation are a product of the

423 implicit learning system; either suppressing alternative learning mechanisms which may be

424 able to overcome persisting errors (Shmuelof et al., 2012; Vaswani et al., 2015) and/or

425 modulating the system's sensitivity to errors dependent on their size, variability and history

426 (Albert et al., 2021).

427 One factor that may explain the discrepancy between our results and those of Langsdorf

428 et al. (2021), is the level of experimental control. Our study was conducted online, with

429 participants completing the task unsupervised at home, whereas Langsdorf et al. (2021)

430 conducted a laboratory-based study. The disparities associated with these environments

431 may have contributed to small but significant differences in motivation, attention and

432 behaviour. That said, our data are typical of many previous lab-based visuomotor

433 adaptation studies, with comparable levels of final performance (Huberdeau et al., 2015;

434 Morehead, Qasim, Crossley, \& Ivry, 2015; Vaswani et al., 2015; Neville \& Cressman, 2018;

435 Weightman et al., 2020; Weightman, Brittain, Miall, \& Jenkinson, 2021). Furthermore, initial

436 evidence suggests that kinematic data and results from online motor learning studies are

437 analogous with those collected in a controlled lab environment (Tsay, Lee, Ivry, \& Avraham,

438 2021) and that online tasks perform well in terms of accuracy and precision with regards to

439 timing of visual stimuli and response capture (Bridges, Pitiot, MacAskill, \& Peirce, 2020).

440 An additional difference between the two studies, which may have had some bearing on

441 the results, is the nature of response cueing used. We used a visual countdown sequence 
which enabled participants to accurately synchronise their movements with the go-signal and ensured a tight coupling between response times and the preparation time groupings. In contrast, Langsdorf et al. (2021) displayed the target and instructed participants to wait until they heard a tone before responding. This difference in protocol resulted in response times closer to 3 seconds. One may argue that more than 2.5 seconds are required to fully prepare for a $45^{\circ}$ rotated reach. This is unlikely however, as we show that even a $30^{\circ}$ angle was not fully compensated, and there was no evidence for a relationship with the preparation interval for those groups. Previous research has also shown that mental rotation and aiming towards angles up to $90^{\circ}$ can be achieved in $\sim 1$ second (McDougle $\&$

451 Taylor, 2019). The modelling data additionally supports this, as it assumes that mental rotation to the target angle is achieved within the allotted preparation time for most conditions (with the eventual reach angle reduced by the learning ratio). There is perhaps an argument to suggest that attending to the countdown sequence may have interfered with mental rotation and other cognitive planning processes, by, for instance vying for attentional resources and thus contributing to a consistent reach undershoot (Taylor \& Thoroughman, 2007; Galea, Sami, Albert, \& Miall, 2010). Nevertheless, prior studies using auditory response cueing have not cited attention to the timed-response sequence as a potential confound (Haith et al., 2015; Leow, Gunn, Marinovic, \& Carroll, 2017; McDougle \& Taylor, 2019).

Despite participants failing to eliminate residual errors during late adaptation, our data do include some hallmarks of a speed-accuracy trade-off. We found that motor adaptation was impaired in the $45^{\circ}$ and $60^{\circ}$ rotational groups, when planning time was restricted to 0.35 seconds. This result is in line with previous studies who report reduced error compensation when preparation times are limited (Fernandez-Ruiz et al., 2011; Leow, Gunn, et al., 2017; 
Leow, Marinovic, Riek, \& Carroll, 2017; Albert et al., 2021) and may reflect the suppression restricted preparation times was coupled with an increased retention during no-feedback errors are approximately equal, because the preparation time exceeded that required for mental rotation. However, these assumptions should be treated with some caution after recent commentary on how the dichotomy of implicit and explicit components of motor adaptation are inferred (Hadjiosif \& Krakauer, 2021). $60^{\circ}$ rotational group (Figure. 3). On average, there was a linear increase in learning asymptote as preparation time increased, which may reflect how mental rotation of the intended movement angle increases the accuracy of reaches (McDougle \& Taylor, 2019; asymptotic differences, until statistically significant, yet data from our other rotational groups and previous literature would suggest that errors would still persist. To achieve full compensation, it is possible that explicit information about the nature of rotation ought to be provided (McDougle \& Taylor, 2019) or strategies primed using methods such as verbal aiming reports (Bond \& Taylor, 2015; Brudner, Kethidi, Graeupner, Ivry, \& Taylor, 2016; 
490 In summary, our data suggests that extending motor preparation and planning periods

491 alone is insufficient to eliminate residual errors during visuomotor adaptation, irrespective

492 of the size of imposed cursor rotation. While increased preparation time may help to

493 improve error reduction at larger rotation magnitudes, our results suggest there remains a

494 limit at which learning saturates at asymptote, perhaps only overcome with priming of

495 explicit strategies, further instruction or changes to experimental variables. Understanding

496 why the central nervous system fails to fully adapt movements in response to

497 environmental changes may be key when aiming to optimise rehabilitation protocols

498 following brain injury or disease. 


\section{References}

501 Albert, S. T., Jang, J., Sheahan, H. R., Teunissen, L., Vandevoorde, K., Herzfeld, D. J., \& 502 Shadmehr, R. (2021). An implicit memory of errors limits human sensorimotor adaptation.

503 Nature human behaviour, 1-15. doi: https://doi.org/10.1038/s41562- 020-01036-x

504 Anguera, J. A., Reuter-Lorenz, P. A., Willingham, D. T., \& Seidler, R. D. (2010). Contributions 505 of spatial working memory to visuomotor learning. Journal of cognitive neuroscience, 22(9), 506 1917-1930. doi: https://doi.org/10.1162/jocn.2009.21351

507 Bhat, R. B., \& Sanes, J. N. (1998). Cognitive channels computing action distance and

508 direction. Journal of Neuroscience, 18(18), 7566-7580. doi:

509 https://doi.org/10.1523/JNEUROSCI.18-18-07566.1998

Bond, K. M., \& Taylor, J. A. (2015). Flexible explicit but rigid implicit learning in a visuomotor adaptation task. Journal of neurophysiology, 113(10), 3836-3849. doi: https://doi.org/10.1152/jn.00009.2015

513 Bridges, D., Pitiot, A., MacAskill, M. R., \& Peirce, J. W. (2020). The timing mega-study:

514 comparing a range of experiment generators, both lab-based and online. PeerJ, 8, e9414.

516 Brudner, S. N., Kethidi, N., Graeupner, D., Ivry, R. B., \& Taylor, J. A. (2016). Delayed feedback during sensorimotor learning selectively disrupts adaptation but not strategy use. Journal of neurophysiology, 115(3), 1499-1511. doi: https://doi.org/10.1152/jn.00066.2015

519 Cunningham, H. A. (1989). Aiming error under transformed spatial mappings suggests a 520 structure for visual-motor maps. Journal of experimental psychology: Human perception and performance, 15(3), 493. doi: https://doi.org/10.1037/0096- 1523.15.3.493

522 Fernandez-Ruiz, J., Wong, W., Armstrong, I. T., \& Flanagan, J. R. (2011). Relation between 523 reaction time and reach errors during visuomotor adaptation. Behavioural brain research, 524 219(1), 8-14. doi: https://doi.org/10.1016/j.bbr.2010.11.060

525 Galea, J., Sami, S., Albert, N., \& Miall, R. C. (2010). Secondary tasks impair adaptation to 526 step-and gradual-visual displacements. Experimental brain research, 202(2), 473-484. doi: 10.1007/s00221-010-2158-x

528

529

530

531

532
Georgopoulos, A. P., Lurito, J. T., Petrides, M., Schwartz, A. B., \& Massey, J. T. (1989). Mental rotation of the neuronal population vector. Science, 243(4888), 234-236. doi:

https://doi.org/10.1126/science.2911737

Georgopoulos, A. P., \& Massey, J. (1987). Cognitive spatial-motor processes. Experimental brain research, 65(2), 361-370. doi: https://doi.org/10.1007/BF00236309

Hadjiosif, A. M., \& Krakauer, J. W. (2021). The explicit/implicit distinction in studies of visuomotor learning: Conceptual and methodological pitfalls. European Journal of Neuroscience, 53(2), 499-503. doi: https://doi.org/10.1111/ejn.14984 
536

Haith, A. M., Huberdeau, D. M., \& Krakauer, J. W. (2015). The influence of movement preparation time on the expression of visuomotor learning and savings. Journal of neuroscience, 35(13), 5109-5117. doi: https://doi.org/10.1523/JNEUROSCI.3869-14.2015

Heuer, H., \& Hegele, M. (2008). Adaptation to visuomotor rotations in younger and older adults. Psychology and aging, 23(1), 190. doi: https://doi.org/10.1037/0882- 7974.23.1.190

Huberdeau, D. M., Haith, A. M., \& Krakauer, J. W. (2015). Formation of a long-term memory for visuomotor adaptation following only a few trials of practice. Journal of neurophysiology, 114(2), 969-977. doi: https://doi.org/10.1152/jn.00369.2015

Kording, K. P., Tenenbaum, J. B., \& Shadmehr, R. (2007). The dynamics of memory as a consequence of optimal adaptation to a changing body. Nature neuroscience, 10(6), 779786. doi: https://doi.org/10.1038/nn1901

Krakauer, J. W., Ghilardi, M.-F., \& Ghez, C. (1999). Independent learning of internal models for kinematic and dynamic control of reaching. Nature neuroscience, 2(11), 1026. doi: https://doi.org/10.1038/14826

Krakauer, J. W., Pine, Z. M., Ghilardi, M.-F., \& Ghez, C. (2000). Learning of visuomotor transformations for vectorial planning of reaching trajectories. Journal of Neuroscience, 20(23), 8916-8924. doi: https://doi.org/10.1523/JNEUROSCI.20- 23-08916.2000

Langsdorf, L., Maresch, J., Hegele, M., McDougle, S. D., \& Schween, R. (2021). Prolonged response time helps eliminate residual errors in visuomotor adaptation. Psychonomic Bulletin \& Review, 1-11. doi: https://doi.org/10.3758/s13423-020-01865-x

Leow, L.-A., Gunn, R., Marinovic, W., \& Carroll, T. J. (2017). Estimating the implicit component of visuomotor rotation learning by constraining movement preparation time. Journal of neurophysiology, 118(2), 666-676. doi: https://doi.org/10.1152/jn.00834.2016

Leow, L.-A., Marinovic, W., Riek, S., \& Carroll, T. J. (2017). Cerebellar anodal tdcs increases implicit learning when strategic re-aiming is suppressed in sensorimotor adaptation. PLOS One, 12(7), e0179977. doi: https://doi.org/10.1371/journal.pone.0179977

Lurito, J., Georgakopoulos, T., \& Georgopoulos, A. (1991). Cognitive spatial- motor processes. Experimental Brain Research, 87(3), 562-580. doi:

https://doi.org/10.1007/BF00227082

Mazzoni, P., \& Krakauer, J. W. (2006). An implicit plan overrides an explicit strategy during visuomotor adaptation. Journal of neuroscience, 26(14), 3642-3645. doi: https://doi.org/10.1523/JNEUROSCI.5317-05.2006

McDougle, S. D., Bond, K. M., \& Taylor, J. A. (2015). Explicit and implicit processes constitute the fast and slow processes of sensorimotor learning. Journal of Neuroscience, 35(26), 9568-9579. doi: https://doi.org/10.1523/JNEUROSCI.5061- 14.2015 
571 McDougle, S. D., \& Taylor, J. A. (2019). Dissociable cognitive strategies for sensorimotor

572 learning. Nature communications, 10(1), 1-13. doi: https://doi.org/10.1038/s41467-018-

573 07941-0

574 Morehead, J. R., Qasim, S. E., Crossley, M. J., \& Ivry, R. (2015). Savings upon re-aiming in

575 visuomotor adaptation. Journal of neuroscience, 35(42), 14386-14396.

576 Neville, K.-M., \& Cressman, E. K. (2018). The influence of awareness on explicit and implicit

577 contributions to visuomotor adaptation over time. Experimental brain research, 236(7),

578 2047-2059. doi: https://doi.org/10.1007/s00221-018-5282-7

579 Peirce, J., Gray, J. R., Simpson, S., MacAskill, M., Höchenberger, R., Sogo, H., Kastman, E., 580 Lindel $\varnothing \mathrm{V}$, J. K. (2019). Psychopy2: Experiments in behavior made easy. Behavior Research 581 Methods, 51(1), 195-203. doi: https://doi.org/10.3758/s13428-018-01193-y

582 Pellizzer, G., \& Georgopoulos, A. P. (1993). Common processing constraints for visuomotor 583 and visual mental rotations. Experimental Brain Research, 93(1), 165-172. doi:

584 https://doi.org/10.1007/BF00227791

585 Shepard, R. N., \& Metzler, J. (1971). Mental rotation of three-dimensional objects. Science, 586 171(3972), 701-703. doi: https://doi.org/10.1126/science.171.3972.701

587 Shmuelof, L., Huang, V. S., Haith, A. M., Delnicki, R. J., Mazzoni, P., \& Krakauer, J. W. (2012). Overcoming motor "forgetting" through reinforcement of learned actions. Journal of 589 Neuroscience, 32(42), 14617-14621a. doi: https://doi.org/10.1523/JNEUROSCI.2184$590 \quad 12.2012$

591 Smith, M. A., Ghazizadeh, A., \& Shadmehr, R. (2006). Interacting adaptive processes with different timescales underlie short-term motor learning. PLoS biology, 4(6). doi:

593 10.1371/journal.pbio.0040179

594 Taylor, J. A., \& Ivry, R. B. (2011). Flexible cognitive strategies during motor learning. PLoS

595 computational biology, 7(3). doi: 10.1371/journal.pcbi.1001096

596 Taylor, J. A., Krakauer, J. W., \& Ivry, R. B. (2014). Explicit and implicit contributions to 597 learning in a sensorimotor adaptation task. Journal of Neuroscience, 34(8), 3023- 3032. doi: 598 https://doi.org/10.1523/JNEUROSCI.3619-13.2014

599 Taylor, J. A., \& Thoroughman, K. A. (2007). Divided attention impairs human motor 600 adaptation but not feedback control. Journal of neurophysiology, 98(1), 317-326. doi: 601 https://doi.org/10.1152/jn.01070.2006

602 Tsay, J. S., Lee, A. S., Ivry, R. B., \& Avraham, G. (2021). Moving outside the lab: The viability 603 of conducting sensorimotor learning studies online. bioRxiv. doi:

604 https://doi.org/10.1101/2021.01.30.181370 
605

606

607

608

609

610

611

612

613

614

615

616

617

618

619

620

621

622

623

624

625

626

627

628

629
Tseng, Y.-w., Diedrichsen, J., Krakauer, J. W., Shadmehr, R., \& Bastian, A. J. (2007). Sensory prediction errors drive cerebellum-dependent adaptation of reaching. Journal of neurophysiology, 98(1), 54-62. doi: https://doi.org/10.1152/jn.00545.2007

van der Kooij, K., Brenner, E., van Beers, R. J., \& Smeets, J. B. (2015). Visuomotor adaptation: how forgetting keeps us conservative. PLoS One, 10(2), e0117901. doi: https://doi.org/10.1371/journal.pone.0117901

Vaswani, P. A., Shmuelof, L., Haith, A. M., Delnicki, R. J., Huang, V. S., Mazzoni, P., Shadmehr, R., Krakauer, J. W. (2015). Persistent residual errors in motor adaptation tasks: reversion to baseline and exploratory escape. Journal of Neuroscience, 35(17), 6969- 6977. doi: https://doi.org/10.1523/JNEUROSCI.2656-14.2015

Voets, S. H., Panouilleres, M. T., \& Jenkinson, N. (2020). An implicit plan still overrides an explicit strategy during visuomotor adaptation following repetitive transcranial magnetic stimulation of the cerebellum. Experimental Results, 1. doi:

https://doi.org/10.1017/exp.2020.16

Vyas, S., O'Shea, D. J., Ryu, S. I., \& Shenoy, K. V. (2020). Causal role of motor preparation during error-driven learning. Neuron, 106(2), 329-339. doi:

https://doi.org/10.1016/j.neuron.2020.01.019

Weightman, M., Brittain, J.-S., Miall, R. C., \& Jenkinson, N. (2021). Direct and indirect effects of cathodal cerebellar tdcs on visuomotor adaptation of hand and arm movements.

Scientific Reports, 11(1), 1-10. doi: https://doi.org/10.1038/s41598- 021-83656-5

Weightman, M., Brittain, J.-S., Punt, D., Miall, R. C., \& Jenkinson, N. (2020). Targeted tdcs selectively improves motor adaptation with the proximal and distal upper limb. Brain Stimulation, 13(3), 707-716. doi: https://doi.org/10.1016/j.brs.2020.02.013

Wilterson, S. A., \& Taylor, J. A. (2019). Implicit visuomotor adaptation remains limited after several days of training. BioRxiv, 711598. doi: https://doi.org/10.1101/711598 


\section{Acknowledgements}

632 This work was funded by the MRC-Versus Arthritis Centre for Musculoskeletal Ageing

633 Research (CMAR). RCM was also partly funded by the Royal Society, Leverhulme and

634 Wellcome Trust.

635

636 Author Contributions

$637 \mathrm{MW}, \mathrm{JSB}$ and NJ conceived and designed the study with input from RCM. MW collected the

638 data. MW, JSB, RCM and NJ analysed and interpreted the data. MW wrote the first draft of

639 the paper. JSB, RCM and NJ reviewed and edited the paper and all authors agreed with the

640 final submitted version.

641 The authors declare no competing interests. 